\title{
Introduction to the thematic issue on Challenges in Engineering Intelligent Environments
}

\author{
Asier Aztiria ${ }^{\mathrm{a}}$, Aaron Crandall ${ }^{\mathrm{b}}$ and Gordon Hunter ${ }^{\mathrm{c}, *}$ \\ ${ }^{a}$ Mondragon Unibertsitatea, Spain \\ ${ }^{\mathrm{b}}$ Washington State University, USA \\ ${ }^{\mathrm{c}}$ Kingston University, $U K$
}

\section{Introduction}

This issue of JAISE is focused on the theme of Challenges in Engineering Intelligent Environments. Since the inception of ubiquitous computing, the difficulties in deploying computing systems in our everyday environments have been recognized. Computing resources, electrical power consumption, visibility, networking, wireless tools, user interfaces, ad hoc infrastructures, security and other issues of concern have long been targets of continued research and engineering efforts.

Intelligent Environments have been strong candidates for bringing limited prototype/pilot versions of the ubiquitous computing concept into everyday use. Applications in the fields of healthcare, automation, education, energy efficiency, and working environments have all seen notable successes. As 'big data' and large scale analytics have been brought to bear on these types of tools, new issues relating to data storage, machine learning, and privacy have been exposed.

The research community targeting these fields has repeatedly sought high quality engineering solutions to problems arising in smart environments. This thematic issue was proposed and compiled to bring together exemplars of problems engineering teams face, the research approaches to building better smart environments, and to enhance awareness of how these problems might be overcome. The editors have sought out papers in areas including: middleware, software engineering, machine learning, sensors and systems, and user experience to demonstrate the diversity of

*Corresponding author. E-mail: G.Hunter@kingston.ac.uk. methodologies and technologies being applied to solve smart environment problems.

With the new push towards achieving the 'Internet of Things' (IoT) paradigm, addressing and surmounting these issues become ever more important. The Guest Editors of this JAISE thematic issue, Gordon Hunter, Aaron Crandall and Asier Aztiria, have sought to make this a relevant, timely and interesting collection of articles for the Ambient Intelligence and Smart Environments community. We cannot show the full scope of the work being done to overcome engineering challenges in smart environments in a single issue of the journal, and we look forward to hearing from readers about other areas and problems that need addressing if the field is to truly bring smart environments to achieve their full promise and potential in our daily lives.

\section{In this thematic issue}

In the paper 'Managing Home Care with Loose Goals and Policies', the focus is on the goals and policies that are used for managing systems in a flexible way without requiring specialised technical knowledge. The authors present an approach to automated home care management that considers loose goals and policies which accommodate probabilistic interpretations and analysis of system inputs/outputs and fuzziness in rules.

The paper 'DOMeMan: A Holistic Framework for Digital Object Memories' introduces the concept of digital object memories (DOMe), that enhance the 
IoT concept by attaching a virtual storage space to each physical object. This allows the collection of all object-related information generated along the entire life-cycle chain of this object. In this paper, the authors present a framework based on a structured data model and a set of tools to create new, and to migrate existing, applications to digital object memories.

The paper entitled 'Managing Adaptive Spoken Dialogue for Intelligent Environments' presents HIS-OwlSpeak, a model-driven dialogue manager for Intelligent Environments. The authors consider different modes of dialogue control, rule-based and probabilistic, for internal adaptivity. For these, a Hidden Information State (HIS) approach, featuring inherent handling of uncertainty in dialogue systems, is applied to a model-driven dialogue manager.

In 'Learning Distributed Deployment and Configuration Trade-offs for Context-Aware Applications in Intelligent Environments', the authors pro- pose a modular design philosophy for smart IoT applications that enables a more optimal deployment of such systems. The authors present and validate a methodology to inspect and learn the trade-offs of different deployment schemes for IoT applications, in order to allow autonomous optimization of their configurations.

\section{Acknowledgements}

The guest editors wish to thank all the reviewers for their valuable contributions to this thematic issue. Additionally, we gratefully acknowledge the support and guidance of the editorial staff of JAISE. Finally, we would like to express our great appreciation to all the authors for their high-quality submissions that made this thematic issue possible. 